\title{
Extraction and identification of platelet-derived microparticles
}

\author{
JUN GUO $^{1}$, CAN FENG ${ }^{2}$, BILI ZHANG ${ }^{3}$, SHIYANG ZHANG ${ }^{1}$, \\ XIAXIAN SHEN $^{3}$, JIAQI ZHU ${ }^{3}$ and XIAN-XIAN ZHAO ${ }^{3}$ \\ ${ }^{1}$ Department of Geriatrics, Anhui Provincial Hospital, Hefei, Anhui 230000; \\ ${ }^{2}$ Department of Cardiology, Yueyang Hospital of Integrated Traditional Chinese and Western Medicine, \\ Shanghai University of Traditional Chinese Medicine; ${ }^{3}$ Department of Cardiology, Changhai Hospital, \\ Second Military Medical University, Shanghai 200433, P.R. China
}

Received February 9, 2019; Accepted June 7, 2019

DOI: $10.3892 / \mathrm{mmr} .2019 .10484$

\begin{abstract}
Microparticles are carriers of signals for intracellular signal transduction. These carriers include proteins, mRNAs, microRNAs and other bioactive substances. Platelets are a major source of circulating microparticles, and microparticles are closely associated with the development of certain cardiovascular diseases. In the present study, a method for separating, extracting and identifying platelet-derived microparticles was developed and differences in the expression of surface proteins on microparticles harvested from platelets stimulated by vortexing or treatment with thrombin was investigated. The counts, composition, sizes and inner structures of microparticles were determined using flow cytometry and transmission electron microscopy. Additionally, it was demonstrated that platelets could be readily activated, and a large quantity of microparticles with varying complex compositions, structures and sizes were derived from activated platelets. High purity platelet-derived microparticles were obtained by gradient centrifugation. However, the microparticles derived from platelets stimulated by thrombin treatment or vortexing differed significantly in the levels of CD63. The present study aimed to provide improved options for the extraction and identification of microparticles.
\end{abstract}

\section{Introduction}

Platelets are derived from mature megakaryocytes, and are biconvex in shape, when they have not been activated, have no nuclei and have a diameter of 2-4 $\mu \mathrm{m}$. Platelets have a short lifespan, typically 1-2 weeks. In addition to hemostasis, platelets serve an important role in pathophysiological processes, including angiogenesis, atherosclerosis, tissue regeneration and immune regulation $(1,2)$.

Correspondence to: Professor Xian-Xian Zhao, Department of Cardiology, Changhai Hospital, Second Military Medical University, 168 Changhai Road, Shanghai 200433, P.R. China

E-mail: 13601713431@163.com

Key words: platelets, microparticles, transmission electron microscopy, flow cytometry, surface protein
Platelet-derived microparticles (PMPs) are submicron particles with a diameter of $0.1-1 \mu \mathrm{m}$ that are secreted by activated or apoptotic platelets (3). Particles of $<0.1 \mu \mathrm{m}$ in diameter are called exosomes, while particles $>1 \mu \mathrm{m}$ in diameter are termed apoptotic bodies $(4,5)$. Microparticles have been demonstrated to act as carriers of signals for communication between cells (1). However, because of their small size and the diverse structures and phenotypes of PMPs, there are considerable challenges in handling and characterizing these particles (6).

In the present study, the traditional method of extracting PMPs was improved upon, and differences in the expression of molecular markers on the surface of PMPs were investigated. Transmission electron microscopy (TEM) was used to identify platelets and their microparticles. Platelets were readily activated using a simple protocol, and a large quantity of microparticles of varying compositions, sizes and structures were derived from the activated platelets. A high degree of purity of PMPs was attained through gradient centrifugation, and additionally it was demonstrated that the standard practice of using flow cytometry to identify PMPs may underestimate the actual number of microparticles.

\section{Materials and methods}

Sample acquisition. Platelet-rich plasma (PRP) was obtained from healthy volunteers from the blood station of Changhai Hospital affiliated to The Second Military Medical University between May and December 2015. Volunteers provided informed consent for the collection of blood samples and the protocol used in the present was approved by The Ethics Committee of Changhai Hospital. Blood (200 ml) was collected in the morning at the blood station prior to breakfast from each volunteer. There were 32 volunteers, 23 males and 9 females. The age of volunteers was 20-40 years old. The free-flowing technique using a $16 \mathrm{G}$ needle was employed for blood collection to prevent platelet activation. The initial centrifugation of the PRP, as described below, was performed $<2 \mathrm{~h}$ after collection. Aspirin (0.5 g; Sigma-Aldrich; Merck KGaA) was dissolved in $1 \mathrm{ml}$ DMSO, sterilized by filtration through a bacterial filter $(0.22 \mu \mathrm{m})$, and the filtrate was added to each unit of PRP.

Separation and extraction of PMPs. PRP was dispensed into a $15 \mathrm{ml}$ centrifuge tube and washed using $5 \mathrm{ml}$ of $4.2 \mathrm{mM}$ ethylene 
diamine tetra-acetic acid per tube. The tubes were centrifuged at $150 \mathrm{x} \mathrm{g}$ for $15 \mathrm{~min}$ at $22^{\circ} \mathrm{C}$ and the pellet containing the red blood cells was discarded. The supernatant was further centrifuged at $1,000 \times \mathrm{g}$ for $10 \mathrm{~min}$ at $22^{\circ} \mathrm{C}$ and the supernatant was discarded. The pellet containing the platelets was resuspended in $1 \mathrm{ml}$ of Hepes- $\mathrm{NaCl}_{2}$ buffer $(10 \mathrm{mM}$ HEPES, $0.85 \% \mathrm{NaCl}_{2}, \mathrm{pH} 7.4$ ) and transferred to a $1.5 \mathrm{ml}$ tube. Each tube contained 10-12 million platelets. Thrombin (Sigma-Aldrich; Merck KGaA) was added at a final concentration of $0.1 \mathrm{IU} / \mathrm{ml}$ to tubes to activate the platelets, each tube was mixed gently and evenly, and subsequently placed in a $37^{\circ} \mathrm{C}$ cell incubator for $90 \mathrm{~min}$. Other tubes were vortexed at room temperature for $1 \mathrm{~min}$ (oscillation frequency 2,800 beats/min) to activate platelets. The suspension was centrifuged at 3,200 x $\mathrm{g}$ for $15 \mathrm{~min}$ at $4^{\circ} \mathrm{C}$ and the supernatant was transferred to a new $1.5 \mathrm{ml}$ tube. The supernatant was further centrifuged at 20,000 $\mathrm{x} g$ for $90 \mathrm{~min}$ at $4^{\circ} \mathrm{C}$ and the supernatant was discarded, leaving the pellet which contained the PMPs.

Flow cytometry. Data was analyzed by CellQuest v5.1 software (BD Bioscience). Each tube of PMPs was resuspended in $200 \mu \mathrm{l}$ binding buffer (BD Bioscience) and mixed evenly. Polystyrene microspheres with a diameter of $1 \mu \mathrm{m}$ (Sigma-Aldrich; Merck KGaA) were diluted 1:1,000 with PBS and $5 \mu \mathrm{l}$ was added to each tube. As a control, one tube of $200 \mu \mathrm{l}$ binding buffer with $5 \mu 1$ microspheres was left blank. The solution was then incubated at $4^{\circ} \mathrm{C}$ for $15 \mathrm{~min}$ in the dark with $20 \mu$ l CD63-phycoerythrin (PE; cat. no. 556020; BD Bioscience), CD61-PE (cat. no. 555754; BD Bioscience), CD62P-allophycocyanin(APC; cat.no. 550888; BD Bioscience), CD40L-PE (cat. no. 555702; BD Bioscience), CD41-APC (cat. no. 303710; BioLegend, Inc.) or $5 \mu \mathrm{l}$ Annexin V-FITC antibodies (BD Bioscience).

TEM. Platelets with PMPs were fixed in $1 \mathrm{ml} 2 \%$ paraformaldehyde and $2 \%$ glutaraldehyde at $4^{\circ} \mathrm{C}$ for $6 \mathrm{~h}$. After fixing, the samples were centrifuged at $1,000 \mathrm{x}$ g for $10 \mathrm{~min}$ at $4^{\circ} \mathrm{C}$ and supernatant containing the fixative was discarded. The precipitate was washed five times with PBS and centrifuged again at $1,000 \mathrm{x} \mathrm{g}$ at $4^{\circ} \mathrm{C}$ for $10 \mathrm{~min}$. The precipitate was resuspended in plasma (supernatant obtained from the second centrifugation) and centrifuged at $1,000 \mathrm{x} \mathrm{g}$ at $4^{\circ} \mathrm{C}$ for $30 \mathrm{~min}$. The majority of the supernatant (plasma) was discarded, leaving a small volume. The samples were fixed using $1 \%$ osmium tetroxide at $4^{\circ} \mathrm{C}$ for $2 \mathrm{~h}$, rinsed once with PBS and centrifuged at $1,000 \mathrm{x} \mathrm{g}$ for $10 \mathrm{~min}$ at $4^{\circ} \mathrm{C}$. Gradient dehydration was performed as follows: $70 \%$ acetone for $15 \mathrm{~min}, 80 \%$ acetone for $15 \mathrm{~min}, 90 \%$ acetone for $15 \mathrm{~min}$, and $100 \%$ acetone for $10 \mathrm{~min}(\mathrm{x} 2)$. The pellet was embedded in a transparent capsule no. 3 (Electron Microscopy room of Second Military Medical University) with epoxy resin and oven-dried at $45^{\circ} \mathrm{C}$ for $12 \mathrm{~h}$ and $60^{\circ} \mathrm{C}$ for $36 \mathrm{~h}$. The embedded block was sliced into $70 \mathrm{~nm}$ thick sections with an ultramicrotome and placed on a copper mesh covered with polyvinyl formal film. Melted wax was dripped on to a sterile Petri dish to form a wax plate, a few drops of lead dye solution were dropped on to the wax and the copper mesh with the sample was placed on top of the lead dye droplet and incubated for $15 \mathrm{~min}$ at room temperature. The copper mesh was taken from the lead dye solution, washed three times with distilled water, dried with filter paper and observed by TEM.
The specimens were examined using an electron microscope (HT7700; Hitachi, Ltd.) at an operating voltage of $100 \mathrm{kV}$ (magnification, x1,000-5,000). Images were analyzed using ImageJ v1.8.0 software (National Institutes of Health).

Statistical analysis. SPSS v19.0 statistical software (IBM Corp.) was used for data analysis. All data are expressed as the mean \pm standard error of the mean. A two-tailed Student's $\mathrm{t}$-test was used for statistical analysis. $\mathrm{P}<0.05$ was considered to indicate a statistically significant difference.

\section{Results}

PMPs can be separated by gradient centrifugation. There is no standard protocol to extract PMPs as far as the authors are aware. However, a protocol for the isolation of PMPs from blood samples was recommended (Fig. 1A). In the present study, gradient centrifugation was used to extract PMPs, with some adjustments. During this process, red blood cells and the majority of platelet fragments were removed from the sample and the resulting precipitate containing the PMPs could be observed at the base of the tube (Fig. 1B). However, it should be noted that a portion of microparticles were lost during the gradient centrifugation.

Flow cytometry results of PMPs. The distribution ranges of the particles with different diameters were divided (Fig. 2A): R1 means $1.0 \mu \mathrm{m}$ polystyrene microspheres; R2 means particles smaller than $1.0 \mu \mathrm{m}$; R3 means particles larger than $1.0 \mu \mathrm{m}$. Flow cytometry analysis of PMPs demonstrated that platelets were markedly sensitive to both physical and chemical stimuli. Pretreatment with aspirin effectively reduced the activation of platelets; however, the attenuation of activation was not complete (Fig. 2B and C). PRP without aspirin pretreatment derived a portion of PMPs, which were lost during the gradient centrifugation, then after totally being activated, the PMPs finally obtained were obviously fewer than PRP with aspirin pretreatment (Fig. 2D). Treatment with thrombin or vortexing stimulated the release of a large number of microparticles from the PMPs. The majority of the precipitates obtained by gradient centrifugation contained particles $<1 \mu \mathrm{m}$ in diameter, although it was possible that platelet fragments or large vesicles were also present (Fig. 2E and F).

Labeling with Annexin V or the five platelet surface markers (CD61, CD62P, CD63, CD40L and CD41) demonstrated that the targets were present on the surface of the microparticles at high levels in the PMPs obtained from platelets stimulated by thrombin or vortexing. There was no significant difference in the expression of surface markers found between PMPs from the thrombin-stimulated platelets compared with the vortex-stimulated platelets, except in the case of CD63, which was significantly higher in the PMPs from vortex-activated platelets $(55.38 \pm 5.27 \%$ vs. $43.50 \pm 3.86 \%$; $\mathrm{P}<0.05$; Fig. 3 ).

TEM of platelets and PMPs. Platelets which had not been pretreated with aspirin displayed an increased level of activation after centrifugation compared with aspirin-treated platelets and the untreated platelets possessed extended pseudopodia compared with the aspirin-treated platelets (Fig. 4A and B). The distal portion of the pseudopodia gradually separated from the platelet body to form a membrane chain (Fig. 4C). 

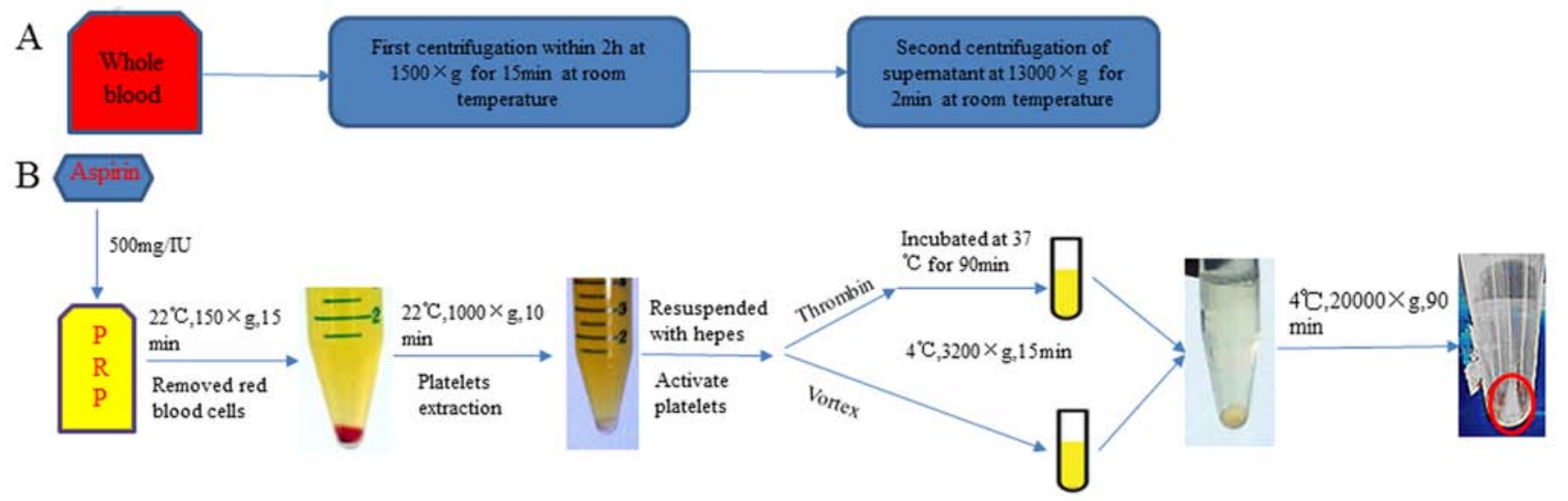

Figure 1. Protocol for extracting and purifying PMPs. (A) The recommend protocol for PMP extraction. (B) Protocol used in the present study for PMPs extraction. The precipitate in the red circle were finally obtained PMPs. PMPs, platelet-derived microparticles; PRP, platelet-rich plasma.
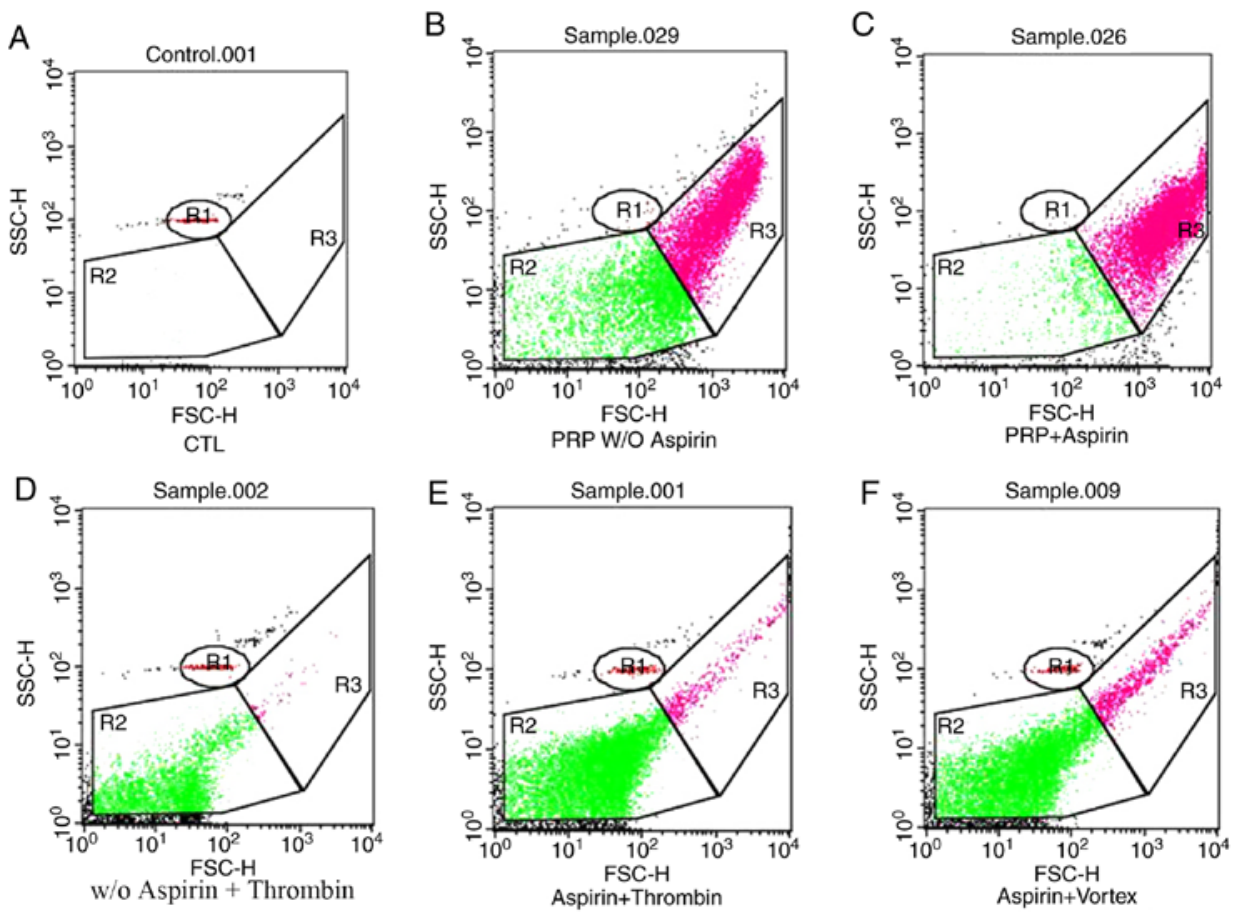

Figure 2. Flow cytometry analysis of purified PMPs. FSC/SSC plots of PMPs. The gate was set such that the upper right limit on the FSC was $1.0 \mu \mathrm{m}$. (A) Control sample. (B) PRP not pre-treated with aspirin after the first centrifugation. (C) PRP pre-treated with aspirin after the first centrifugation. (D) PMPs harvested from PRP not pre-treated with aspirin and stimulated with thrombin. (E) PMPs harvested from PRP pre-treated with aspirin and stimulated with thrombin. (F) PMPs harvested from PRP pre-treated with aspirin and stimulated by vortexing. R1, $1.0 \mu \mathrm{m}$ polystyrene microspheres; R2, $<1.0 \mu \mathrm{m}$ particles; R3, $>1.0 \mu \mathrm{m}$ particles; PMPs, platelet-derived microparticles; PRP, platelet-rich-plasma; FSC, forward scatter; SSC, side scatter; w/o, without.

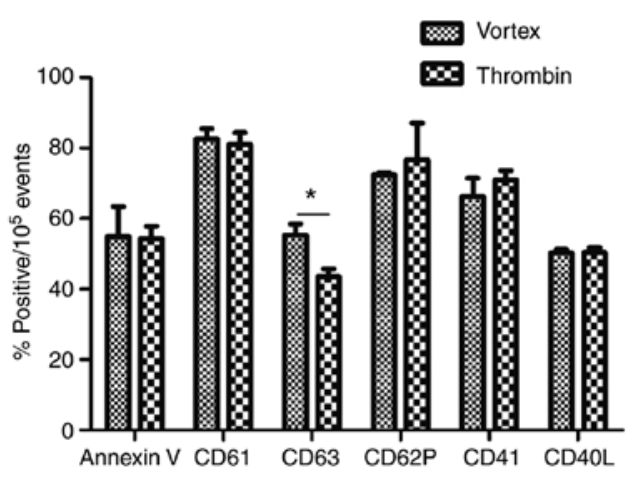

Figure 3. Flow cytometry analysis for marker staining of platelet-derived microparticles stimulated by thrombin or by vortexing. ${ }^{*} \mathrm{P}<0.05$.
Eventually, the membrane chain broke and the microparticles were secreted. Treatment with thrombin and vortexing activated platelets resulting in the release of PMPs (Fig. 4D and E). PMPs have different shapes and complex ultrastructures, and their contents, membrane structure and electron density vary. In a similar way to medicine capsules, PMPs contain biologically active substances such as $\alpha$-granules, glycogen granules and mitochondria (Fig. 4F).

\section{Discussion}

Microparticles secreted by platelets are involved in the regulation of many physiological and pathophysiological processes 

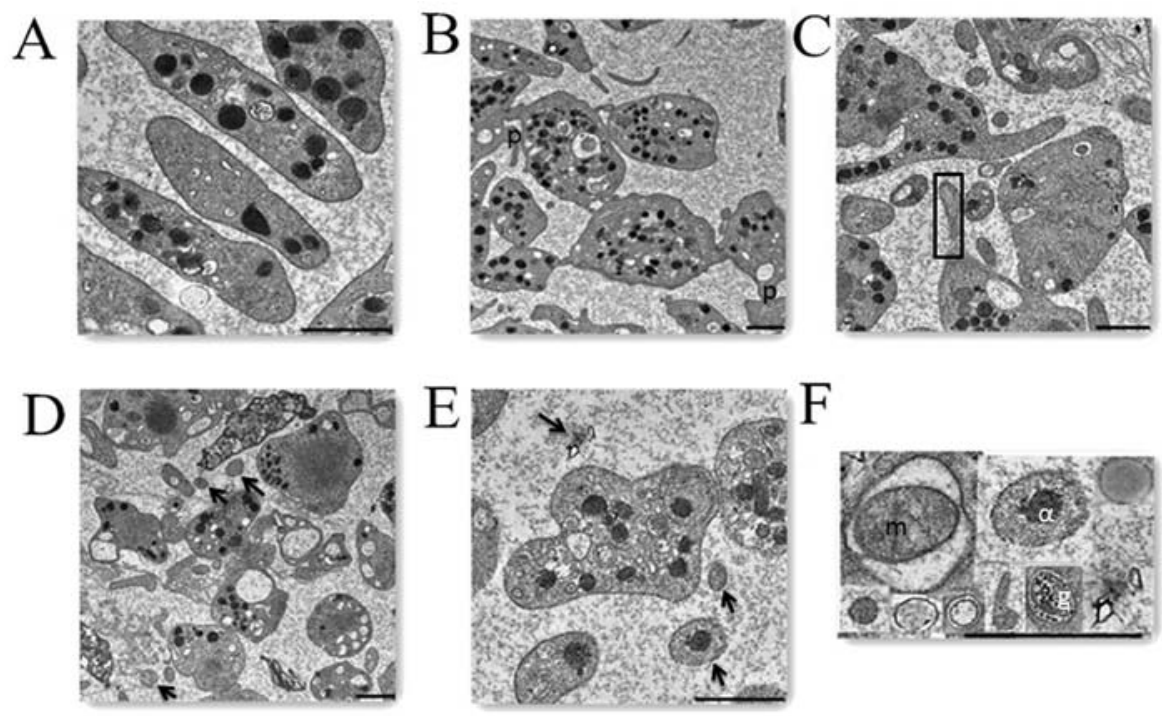

Figure 4. Transmission electron microscopy of platelets and PMPs. (A) Platelets pre-treated with aspirin had relatively stable structures. (B) Platelets not pre-treated with aspirin had extended pseudopodia. (C) A microparticle being released from a platelet (black box). (D) Released PMPs from platelets activated using thrombin. (E) Released PMPs from platelets activated by vortexing. (F) PMPs with different shapes, contents, and membrane structure. The arrows indicate PMPs. Scale bar, $1.0 \mu \mathrm{m}$. PMPs, platelet-derived microparticles. $\alpha, \alpha$-granules; g, glycogen granules; m, mitochondria.

in the body, including coagulation, vasomotor regulation, cell proliferation, differentiation, apoptosis, inflammation, the immune response and the transmission of signals between cells $(1,6)$. Studies have demonstrated that different kinds of cells can secrete microparticles, including red blood cells, lymphocytes, platelets, endothelial cells and tumor cells; however, the microparticles derived by platelets account for $70-90 \%$ of microparticles present in the circulatory system (7-9). Extracting PMPs is a difficult and labor-intensive process. A two-step gradient centrifugation method is recommended to extract PMPs from blood samples, although a standardized approach is not available. In theory, the longer and faster the final centrifugation step, the more particles of smaller sizes can be obtained, although there may be an upper limit. In addition, increasing the number of gradient layers will concurrently increase the purity of the sample, with the caveat that there will also be an increase in the loss of the desired product. Therefore, determining the optimal combination of centrifugal speed, duration and number of gradient layers, to maximize the purity and minimize the loss of microparticles requires further investigation.

In the present study, adjustments were made to the most frequently used two-step gradient centrifugation method. PRP was obtained from the blood station at Changhai Hospital affiliated to The Second Military Medical University, and met China's national quality standards, reducing the proportion of other cells obtained. Residual red blood cells were removed from the PRP in the first centrifugation step, further improving the purity of the platelets. The precipitation of the platelets was achieved by centrifugation, after which the platelets were activated using thrombin or by vortexing. To increase the purity of PMPs further, two additional centrifugation steps were used. However, there was still a small proportion of unwanted particles $>1 \mu \mathrm{m}$ in diameter in the final precipitate, possibly platelet fragments or large vesicles. PMPs are particles with a 0.1-1 $\mu \mathrm{m}$ diameter that are secreted by activated or apoptotic platelets (3). Therefore, a $1 \mu \mathrm{m}$ diameter was set as the upper limit for microparticles in the flow cytometry experiments performed in the present study. Platelets can be activated with ease, with activation resulting in the release of a large number of microparticles (10). Platelets continue to release microparticles when they are inactive, although at a lower level (10). Previous studies have demonstrated that collagen, thrombin, lipopolysaccharide, viruses, immune complexes, temperature changes and shear stress could all activate platelets, resulting in the release of microparticles (7,11-15). To minimize the loss of microparticles during transportation and centrifugation, antiplatelet activation or anticoagulant drugs can be added before further activation of platelets, of which aspirin is probably the most economical (16). A previous clinical study reported that aspirin significantly reduced the number of PMPs in circulation in patients with coronary heart disease (17). The present study additionally demonstrated that although aspirin did not completely prevent platelets from being activated, it markedly inhibited the secretion of PMPs. In the PRP samples not pre-treated with aspirin, there was a notable increase in the release of microparticles following centrifugation. This fraction of microparticles was discarded with the supernatant during the gradient centrifugation, and the final number of microparticles extracted was markedly decreased compared with the aspirin pre-treated samples.

Murphy and Gardner (18) studied the effects of temperature on platelets and demonstrated that platelets maintained in vitro at $22^{\circ} \mathrm{C}$ had an improved structure and function. Bode and Knupp (19) demonstrated that platelets lost more glycoproteins and formed more microparticles at $4^{\circ} \mathrm{C}$. Therefore, platelets in the present study were extracted at $22^{\circ} \mathrm{C}$ and the final ultracentrifugation step was performed at $4^{\circ} \mathrm{C}$ to obtain the PMPs.

The mechanism of microparticle production is not fully understood and may be related to the asymmetric loss of proteasomes and membrane phospholipids $(20,21)$. A previous 
study demonstrated that during normal blood flow, activated platelets form a membrane chain downstream of the blood flow, and eventually the membrane chain breaks, releasing microparticles (22). In the present study, platelets were observed to form a membrane chain following activation. Eventually the membrane chain broke and microparticles were released. There are a number of methods used to identify PMPs and their associated markers. After activation, the intracellular calcium concentration of platelets is elevated, and non-selective ion channels on the cell surface and on the mitochondria, in addition to the stimulation of some enzymes, promote the flipping of phosphatidylserine; when present on the extracellular facing side of the cell membrane, phosphatidylserine subsequently acts as a signal for phagocytosis on apoptotic cells (23-25). Therefore, Annexin V is commonly used as a marker for detecting microparticles. However, studies have shown that $50 \%$ of the microparticles in circulation do not present phosphatidylserine on their surface (26). The use of Annexin V alone as a marker of microparticles may, therefore, underestimate the number of microparticles present (26). In the present study, Annexin $\mathrm{V}$ positive particles accounted for $\sim 50 \%$ of the particles obtained. Therefore, the use of Annexin $\mathrm{V}$ alone as a marker for microparticles may be inadequate and may result in a large underestimation of the number of microparticles.

A limitation of using flow cytometry to detect microparticles is the accuracy of detection for sub-200 nm particles (27). TEM is the most accurate and reliable method for identifying microparticles; however, the preparation of samples is a time-consuming process and requires high quality specimens. In order to determine whether the extracted microparticles are derived from platelets, it is also necessary to identify platelet surface-specific molecular markers. In the present study, CD41, CD61, CD62P, CD63 and CD40L were used in combination with Annexin $\mathrm{V}$ to perform a single step detection of microparticles from platelets activated by vortexing or thrombin treatment. Differences in handling and storage methods may result in changes to the surface markers present on PMPs $(28,29)$. The present results demonstrated that all six markers were expressed on the surface of PMPs, with CD63 expression found to be significantly higher in microparticles derived from vortex-stimulated platelets compared with microparticles from thrombin-stimulated platelets. In a previous study, CD63 was used as a biomarker for the detection of exosomes (30). However, in the present study, exosomes were not able to be detected by flow cytometry. Brisson et al (31) demonstrated that the majority of larger extracellular vesicles, up to $1 \mu \mathrm{m}$ in diameter, also expressed CD63. Whether the difference in CD63 levels on PMPs obtained from the two different stimulation methods was a result of differences in the presence of large vesicles is unknown and requires further study. Yuana et al (32) studied microparticles in fresh plasma using electron microscopy. The results demonstrated that microparticles existed in various shapes, including round, drop-shaped, tubular and cup-shaped. In the present study, based on the results of TEM, PMPs also displayed a variety of shapes, sizes, contents, ultrastructure and electron densities. After activation, platelets secrete particles and tend to disintegrate (33). In the present study, the diameter of microparticles was $200-600 \mathrm{~nm}$. Other differences observed in the TEM images include the presence of either a single or double layered membrane, $\alpha$-granule content, the presence of glycogen granules and the presence of mitochondria. The majority of the particles observed were circular, oval or almost round. The size of microparticles is associated with their contents. Microparticles containing organelles typically have a larger diameter and irregularly shaped particles may be a result of the handling process $(32,33)$. To the best of our knowledge, a certain shape or size of PMP has not been attributed to a particular function. Difficulties in isolating specific types of PMPs has hampered progress in understanding differences in function.

In conclusion, high purity PMPs may be obtained by gradient centrifugation, although a small fraction of platelet fragments or large vesicles may remain. A higher purity of PMPs can be achieved if a $1 \mu \mathrm{m}$ filter is used. At present, the use of flow cytometry to detect PMPs based on Annexin V may lead to inaccurate results. TEM is more accurate in identifying microparticles; however, the technical limitations, labor-intensive preparation process and considerably lower throughput make TEM less convenient. Determining the best method to use towards identifying PMPs may be best decided on a per case basis; it may be possible to use TEM on a small sample of purified PMPs to confirm the results of flow cytometry. Difficulties in identifying PMPs may be a result of the diversity of PMPs, and this diversity may additionally underlie the range of functions attributed to PMPs. Therefore, further studies are required to elucidate the function of PMPs and to improve the methods for their identification.

\section{Acknowledgements}

The authors would like to thank $\mathrm{Dr} \mathrm{Li} \mathrm{Su}$ (College of Pharmacy, Second Military Medical University) and Dr Xiao-Yan Fan (Second Military Medical University) for their contributions to flow cytometry and TEM.

\section{Funding}

The present study was supported by a grant from The National Natural Science Foundation of China (grant no. 81570208).

\section{Availability of data and materials}

All data generated and/or analyzed in the present study is included in the published article.

\section{Authors' contributions}

$\mathrm{JZ}, \mathrm{XXZ}, \mathrm{BZ}$ and JG conceived and designed the experiments. JG, CF and XS performed the experiments. JG and SZ analyzed the data and wrote the manuscript. All authors read and approved the final manuscript

\section{Ethics approval and consent to participate}

Patients provided informed consent for the collection of blood samples and the protocol used in the present was approved by the Ethics Committee of Changhai Hospital. 


\section{Patient consent for publication}

Not applicable.

\section{Competing interests}

The authors declare that they have no competing interests.

\section{References}

1. Burnouf T, Goubran HA, Chou ML, Devos D and Radosevic M Platelet microparticles: Detection and assessment of their paradoxical functional roles in disease and regenerative medicine. Blood Rev 28: 155-166, 2014.

2. Semple JW, Italiano JE Jr and Freedman J: Platelets and the immune continuum. Nat Rev Immunol 11: 264-274, 2011.

3. Clark SR, Thomas CP,Hammond VJ, Aldrovandi M, Wilkinson GW, Hart KW, Murphy RC, Collins PW and O'Donnell VB Characterization of platelet aminophospholipid externalization reveals fatty acids as molecular determinants that regulate coagulation. Proc Natl Acad Sci USA 110: 5875-5880, 2013.

4. Mooberry MJ and Key NS: Microparticle analysis in disorders of hemostasis and thrombosis. Cytometry A 89: 111-122, 2016.

5. Montoro-García S, Shantsila E, Marín F, Blann A and Lip GY Circulating microparticles: New insights into the biochemical basis of microparticle release and activity. Basic Res Cardiol 106 911-923, 2011.

6. Kailashiya J: Platelet-derived microparticles analysis: Techniques, challenges and recommendations. Anal Biochem 546: 78-85, 2018.

7. Burnier L, Fontana P, Kwak BR and Angelillo-Scherrer A: Cell-derived microparticles in haemostasis and vascular medicine. Thromb Haemost 101: 439-451, 2009.

8. Horstman LL and Ahn YS: Platelet microparticles: A wide-angle perspective. Crit Rev Oncol Hematol 30: 111-142, 1999.

9. VanWijk MJ, VanBavel E, Sturk A and Nieuwland R: Microparticles in cardiovascular diseases. Cardiovasc Res 59: 277-287, 2003.

10. Cauwenberghs S, Feijge MA, Harper AG, Sage SO, Curvers J and Heemskerk JW: Shedding of procoagulant microparticles from unstimulated platelets by integrin-mediated destabilization of actin cytoskeleton. FEBS Lett 580: 5313-5320, 2006.

11. Reininger AJ, Heijnen HF, Schumann H, Specht HM, Schramm W and Ruggeri ZM: Mechanism of platelet adhesion to von Willebrand factor and microparticle formation under high shear stress. Blood 107: 3537-3545, 2006.

12. Sims PJ, Faioni EM, Wiedmer T and Shattil SJ: Complement proteins C5b-9 cause release of membrane vesicles from the platelet surface that are enriched in the membrane receptor for coagulation factor Va and express prothrombinase activity. J Biol Chem 263: 18205-18212, 1988.

13. Johnson L, Reade MC, Hyland RA, Tan S and Marks DC: In vitro comparison of cryopreserved and liquid platelets: Potential clinical implications. Transfusion 55: 838-847, 2015.

14. Brown GT and McIntyre TM: Lipopolysaccharide signaling without a nucleus: Kinase cascades stimulate platelet shedding of proinflammatory IL-1 $\beta$-rich microparticles. J Immunol 186 5489-5496, 2011.

15. Boilard E, Paré G, Rousseau M ,Cloutier N, Dubuc I, Lévesque T, Borgeat $\mathrm{P}$ and Flamand L: Influenza virus $\mathrm{H} 1 \mathrm{~N} 1$ activates platelets through FcgammaRIIA signaling and thrombin generation Blood 123: 2854-2863, 2014

16. Pan Y, Liang H, Liu H, Li D, Chen X, Li L, Zhang CY and Zen K: Platelet-Secreted MicroRNA-223 promotes endothelial cell apoptosis induced by advanced glycation end products via targeting the insulin-like growth factor 1 receptor. J Immunol 192: 437-446, 2014.
17. Bulut D, Becker V and Mügge A: Acetylsalicylate reduces endothelial and platelet-derived microparticles in patients with coronary artery disease. Can J Physiol Pharmacol 89: 239-244, 2011.

18. Murphy S and Gardner FH: Effect of storage temperature on maintenance of platelet viability-deleterious effect of refrigerated storage. N Engl J Med 280: 1094-1098, 1969.

19. Bode AP and Knupp CL: Effect of cold storage on platelet glycoprotein 1B and vesiculation. Transfusion 34: 690-696, 1994.

20. Gupta N, Li W, Willard B, Silverstein RL and McIntyre TM: Proteasome proteolysis supports stimulated platelet function and thrombosis. Arterioscler Thromb Vasc Biol 34: 160-168, 2014.

21. Morel O, Jesel L, Freyssinet JM and Toti F: Cellular mechanisms underlying the formation of circulating microparticles. Arterioscler Thromb Vasc Biol 31: 15-26, 2011.

22. Tersteeg C, Heijnen HF, Eckly A, Pasterkamp G, Urbanus RT, Maas C, Hoefer IE, Nieuwland R, Farndale RW, Gachet C, et al: FLow-induced PRotrusions (FLIPRs): A platelet-derived platform for the retrieval of microparticles by monocytes and neutrophils. Circ Res 114: 780-791, 2014.

23. Mattheij NJ, Gilio K, van Kruchten R, Jobe SM, Wieschhaus AJ, Chishti AH, Collins P, Heemskerk JW and Cosemans JM: Dual mechanism of integrin alphaIIb $\beta 3$ closure in procoagulant platelets. J Biol Chem 288: 13325-13336, 2013.

24. Bettache N, Gaffet P, Allegre N, Maurin L, Toti F, Freyssinet JM and Bienvenüe A: Impaired redistribution of aminophospholipids with distinctive cell shape change during $\mathrm{Ca}^{2+}$-induced activation of platelets from a patient with Scott syndrome. $\mathrm{Br}$ J Haematol 101: 50-58, 1998.

25. Choo HJ, Saafir TB, Mkumba L, Wagner MB and Jobe SM: Mitochondrial calcium and reactive oxygen species regulate agonist-initiated platelet phosphatidylserine exposure. Arterioscler Thromb Vasc Biol 32: 2946-2955, 2012.

26. Arraud N, Linares R, Tan S, Gounou C, Pasquet JM, Mornet S and Brisson AR: Extracellular vesicles from blood plasma: Determination of their morphology, size, phenotype and concentration. J Thromb Haemost 12: 614-627, 2014.

27. Nolan JP: Flow cytometry of extracellular vesicles: Potential, Pitfalls, and Prospects. Curr Protoc Cytom 73: 1-16, 2015.

28. Flaumenhaft R, Dilks JR, Richardson J, Alden E, Patel-Hett SR, Battinelli E, Klement GL, Sola-Visner M and Italiano JE Jr: Megakaryocyte-derived microparticles: Direct visualization and distinction from platelet-derived microparticles. Blood 113: 1112-1121, 2009.

29. Rank A, Nieuwland R, Delker R, Köhler A, Toth B, Pihusch V, Wilkowski R and Pihusch R: Cellular origin of platelet-derived microparticles in vivo. Thromb Res 126: e255-e259, 2010.

30. Baranyai T, Herczeg K, Onódi Z, Voszka I, Módos K, Marton N, Nagy G, Mäger I, Wood MJ, El Andaloussi S, et al: Isolation of exosomes from blood plasma: Qualitative and quantitative comparison of ultracentrifugation and size exclusion chromatography methods. PLoS One 10: e0145686, 2015.

31. Brisson AR, Tan S, Linares R, Gounou C and Arraud N: Extracellular vesicles from activated platelets: A semiquantitative cryo-electron microscopy and immuno-gold labeling study. Platelets 28: 263-271, 2017.

32. Yuana Y, Koning RI, Kuil ME, Rensen PC, Koster AJ, Bertina RM and Osanto S: Cryo-electron microscopy of extracellular vesicles in fresh plasma. J Extracell Vesicles: 2, 2013 doi: 10.3402/jev.v2i0.21494.

33. Ponomareva AA, Nevzorova TA, Mordakhanova ER, Andrianova IA, Rauova L, Litvinov RI and Weisel JW: Intracellular origin and ultrastructure of platelet-derived microparticles. J Thromb Haemost 15: 1655-1667, 2017. 